\title{
Myocardial T1 and ECV mapping: how we optimise technical aspects of acquisition
}

\author{
Ricardo Wage*, Peter Gatehouse, Nur Hayati Jasmin, Dudley J Pennell \\ From 19th Annual SCMR Scientific Sessions \\ Los Angeles, CA, USA. 27-30 January 2016
}

\section{Background}

MOLLI techniques for the acquisition of T1 maps in the heart are becoming routine practice at many centres. A wide range of techniques and scanning protocols exist in order to produce reproducible and accurate T1 maps [review in Radiographics, consensus paper James Moon]. However, familiarity and experience using these protocols may be limited outside dedicated CMR units.

Our aim is to present practical experience with a specific T1/ECV scanning protocol. We do not intend to suggest that this or any other protocol is the "correct" one, as this is subject to much ongoing investigation.

\section{Methods}

This work is based on the Siemens prototype WIP 448B and its default protocols for 11 heartbeat variants of MOLLI that have been optimized for typical pre-Gad and post-Gad ranges of $\mathrm{T} 1$ values. We present a mixture of experience at $1.5 \mathrm{~T}$ and $3 \mathrm{~T}$.

Step 1 : Retrospective-gated TruFISP 2D cine of the Mid-LV sax. The retro-gating is quite important in this application to see the diastolic pause in full.

Step 2: By viewing the cine with the trigger-time of each frame, find the LV mid-diastolic timing in milliseconds after R-wave trigger, it may be beneficial to optimise timings for septal stability.

Step 3 : Select the T1 mapping protocol optimised for longer T1 values pre-Gad. If diastolic pause is short, select a lower-resolution version which has a shorter shot duration. Adjust MOLLI acquisition timing to match image acquisition into the middle of the diastolic pause.

Step 4: Set the "adjustments volume" (green box) over the LV (approx $12 \times 12 \times 12 \mathrm{~cm}$ cube). [on VB17 this is normally only a reference frequency adjust not a cardiac shim, use only cardiac shim if available. The "standard"

Royal Brompton Hospital, London, United Kingdom

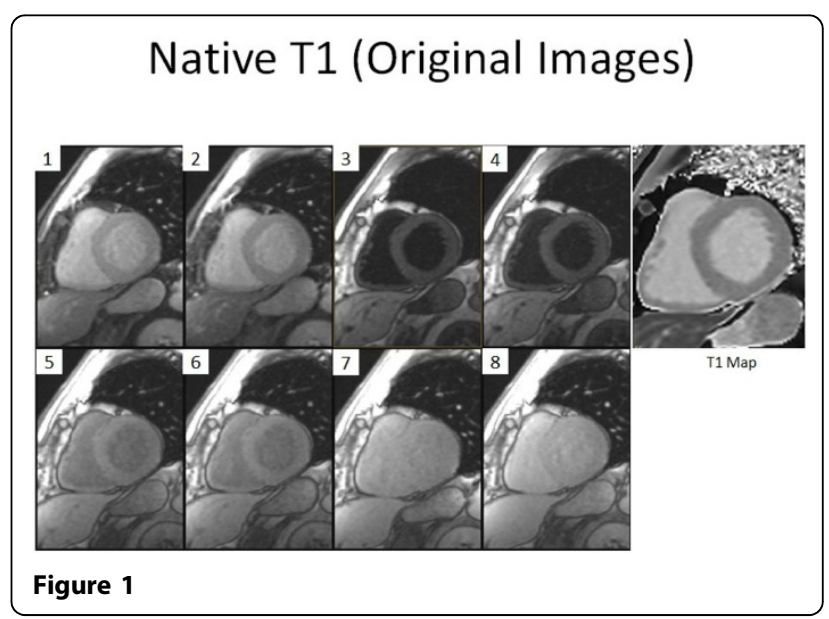

or "advanced" Siemens shim modes are unreliable for cardiac work.

Step 5: Acquire the MOLLI image in Breath-hold 11 cardiac cycles. Monitor ECG during this scan and record any abnormal cardiac cycles or triggers.

Step 6: Image review: This should be performed rigorously during scanning in case re-acquisition is necessary.

Step 7: For Post Contrast study after 14-15 mins of injection. (with altered T1mapping protocol for improved precision in the short-T1 range expected post-Gad). Ensure slice location and timing of shot acquisition are consistent with pre-Gad and post Gad.

\section{Results}

A detailed presentation of one centre's experience with T1/ECV mapping in a variety of challenging patients. Particular emphasis is placed on acquisition as a major pitfall exists that a T1Map is automatically generated from any input images but should be avoided for $\mathrm{T} 1$ measurements without reviewing the underlying image quality. 


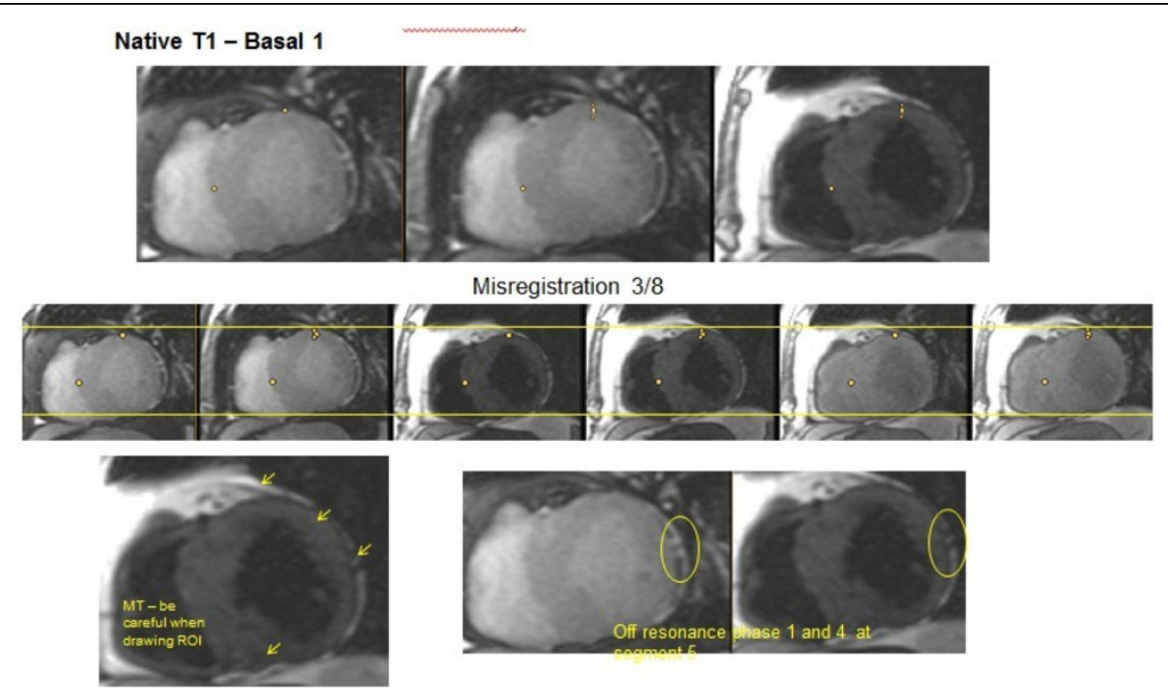

Figure 2

\section{Conclusions}

Familiarity and experience with T1 Mapping may be limited outside of high volume CMR units. Detailed practical guidance to minimize the number of non-diagnostic scans may reduce the "scatter" in $\mathrm{T} 1$ and $\mathrm{ECV}$ measurements, which is currently of large concern for applying these techniques.

Published: 27 January 2016

doi:10.1186/1532-429X-18-S1-T9

Cite this article as: Wage et al:: Myocardial T1 and ECV mapping: how we optimise technical aspects of acquisition. Journal of Cardiovascular Magnetic Resonance 2016 18(Suppl 1):T9.
Submit your next manuscript to BioMed Central and take full advantage of:

- Convenient online submission

- Thorough peer review

- No space constraints or color figure charges

- Immediate publication on acceptance

- Inclusion in PubMed, CAS, Scopus and Google Scholar

- Research which is freely available for redistribution 